units of antitoxin, so that in 36 hours he had had 60,000 units. On the morning of the third day he had developed membrane all over the markedly swollen tonsil and palate, and there was marked edema in the neck. He was given another 36,000 units and the edema was gone and the membrane clearing on the morning of the fourth day. It seems reasonable to me that if 60,000 units given as early in the disease as it is ever possible to give allowed the disease to progress to that extent much smaller doses would have been of doubtful value. The intravenous method will accomplish more. The dosage varies here from 2000 to as high occasionally as 400,000 to 500,000 units.

No patient ever dies when antitoxin is given the first day. Of 431 cases of diphtheria among the employees at the South Department since antitoxin, no deaths have occurred from diphtheria. That ideal result in a period of 15 years is not mere luck. It is just the result that should be reached in all cases instead of the 5 to $10 \%$ mortality of those coming to the hospital. The only way this can be changed is through earlier diagnosis and earlier treatment. The first dose should be as large as the physician can decide is necessary, and intervals of 24 hours should not be allowed to elapse before the next if there is any doubt of its sufflciency. It is difficult to tell at the outset what total dose will be needed and second and third doses may be required. We intend to have no antitoxin given after 48 hours, all that is needed being given so far as possible within that interval and if possible at once.

One word in regard to ill-effects of serum treatment. So striking are the animal experiments of anaphylaxis that every medical student who attends the hospital wonders at one's temerity in giving antitoxin, and the same fear is seen in practice; do we dare to give this or that dose or do we dare to give it at all? It is a surprising peculiarity of the human mind that we fear the infinitesimal danger of serum more than the very great danger of diphtheria toxin.

Personally, I have never seen a death from serum. I have never withheld serum in any case where there was the slightest evidence that it was indicated. Dr. McCollom has stated that in over 40,000 cases at the South Department to whom serum was given, there has never been a death from its use.

The ill effects of serum treatment are:

1. Rashes.

a. Urticaria occurring usually at about the 7-10th day.

$b$. Erythema multiforme occurring about 12-21st day.

2. Fever, practically invariably associated with the efflorescence.

'3. Angio-neurotic edema.

4. Adenopathy.

5. Arthralgia and myalgia.

6. Vomiting, chiefly associated with urticaria and edema.
7. Fainting, invariably in my experience associated with exertion while suffering with serum rash.

8. Shock, very rare and already alluded to.

There is no question that certain people do have a certain susceptibility to serum but from a standpoint of practical medicine, the danger is very slight. You might just as well not give ether. Asthmatics are supposed to be especially unsafe. Recently I saw a patient of Dr. Tobey's, a physician's wife, who had suffered from asthma for years. Dr. Tobey had recently removed polypoid tissue from the nose. During convalescence there was only one mild attack of asthma. While recuperating from the operation, she developed diphtheria. Two doses of 12,000 units were given without any unfavorable effect.

Those subject to horse asthma might not fare so well. We have seen few cases of definite asthma; only two in children. These had no ill effects of serum treatment.

Whenever there is the slightest fear beforehand that serum will prove dangerous, tests may be safely made of susceptibility by injecting an infinitesimal dose and watching for ill effects. If these are absent after a short time the regular dose may be given. If there are symptoms with the minute dose, as soon as they have passed off, a larger dose may be given in the refractory period without fear. This is being extremely cautious in actual practice and would be used only where extreme susceptibility is suspected.

\section{THE SIGNIFICANCE OF SYMPTOMS IN PULMONARY TUBERCULOSIS.*}

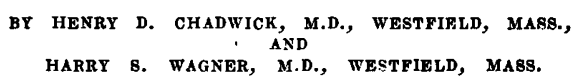

As a rule physicians do not attach enough significance to the early symptoms of pulmonary tuberculosis. In a doubtful case the diagnosis is often delayed until definite physical signs appear in the lungs, or the patient is kept waiting until tubercle bacilli are found in the sputum. We all appreciate how difficult it is, occasionally, even for one giving much time to chest examinations, to find physical signs in the lungs of some patients who have had pulmonary hemorrhages, elevated temperature, or other symptoms indicating tuberculous infection. After a varied period of time many of these patients if left to their own devices develop râles in the apices and other signs of infiltration with the subsequent necrosis, which frees the tubercle hacilli.

When this occurs the patient is past the incipient stage even if the diseased area yet be small in extent. As soon as the bacilli are free the opportunity for extension is greater, and perhaps more important still is the fact that the " Read at the annual meeting of The Massachusetts Medical So. ciety, June 11, 1912. 
viability of the bacilli is enhanced by having a point of exit which allows the discharge of its own metabolic products. These products when encapsulated in a tubercle tend automatically to hinder, or prevent, further growth or bacilli and the progress of the disease.

When we consider that tuberculous infection occurs in nearly all individuals before they are twenty years old, and but seven per cent. die from tuberculosis, we may truly state that it is one of the most curable of all infectious diseases.

But, however, when we study the cases whose natural immunity is insufficient to withstand the infecting bacilli and symptoms are produced of such severity that a diagnosis can be easily made, then we have to deal with one of the most difficult diseases to control, and one in which the mortality yet remains high.

One of the ways in which this mortality may be reduced is by persistent and alert wateh for early symptoms. The symptoms to be looked upon as suspicious are loss of weight, weakness, or undue weariness, frequent or prolonged colds, cough, hemoptysis, pleurisy, increased rapidity of the pulse, and an afternoon elevation of temperature, which is sometimes only present after exertion. One, or several, of these symptoms will be present before bacilli can be found, or in fact before there is any sputum to be examined.

The presence of a cough and positive sputum are two factors which cannot be depended upon if an early diagnosis is desired, as both these signs may be absent even when there is well developed tuberculosis, with marked physical signs in the lungs.

All blood spitting which is not explained by cardiac disease, epistaxis or lobar pneumonia should be .considered good evidence of pulmonary tuberculosis, unless the location of a bleeding point can be found.

To examine a patient after a hemorrhage and tell him that as no physical signs of tuberculosis can be found in the lungs, the blood must have come from the throat, is a mistaken kindness which usually leads to dire results.

The method of handling such patients should be reversed and if a careful examination of nose and throat does not reveal the source of blood, the patient should be told that the bleeding must be within the lungs, and means the beginning of pulmonary tuberculosis. Then the patient can take advantage of his good fortune, which is what an early diagnosis ought to be considered. A hemoptysis is often a "life saver," if the patient happens to consult a doctor who is honest in his statements and one who appreciates his opportunity.

A patient at such times is usually willing to drop business and follow the proper treatment. No other symptom so impresses a person with the gravity of his condition, although, as a matter of fact, it is less serious than weakness or increased rapidity of pulse, which do not attract the patient's attention.
In order to get an idea of the true value of early symptoms in individual cases of pulmonary tuberculosis we have made a careful study of the histories of two hundred cases treated at the Westfield State Sanatorium. One hundred of these cases obtained an arrest of their disease and one hundred died while under treatment or within a few months after discharge. We decided to compare these two groups of patients to see if we could determine what symptoms could be considered favorable, or unfavorable, in forecasting a prognosis or significant in diagnosis.

As the severity of reaction following a tuberculin test is an index of a patient's sensitiveness, it should follow that the immediate symptoms resulting from active tuberculous infection would indicate an individual's natural resistance, or sensitiveness, to the infecting organism. The violence of this reaction would depend upon the natural immunity of the patient and upon the virulence of the infecting strain of bacilli. Other things being equal, early symptoms should indicate the ratio between these two factors.

The onset of these two hundred cases was as follows :

TABLE 1.

Duration of Disease of 100 Cases That Died.

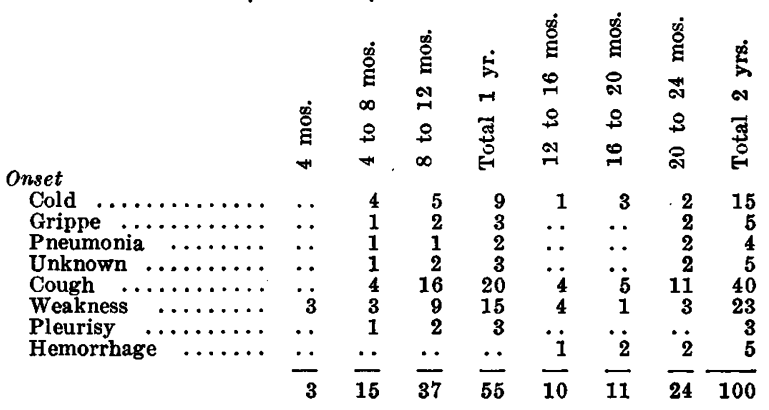

Duration of Disease of 100 Arrested Cases.

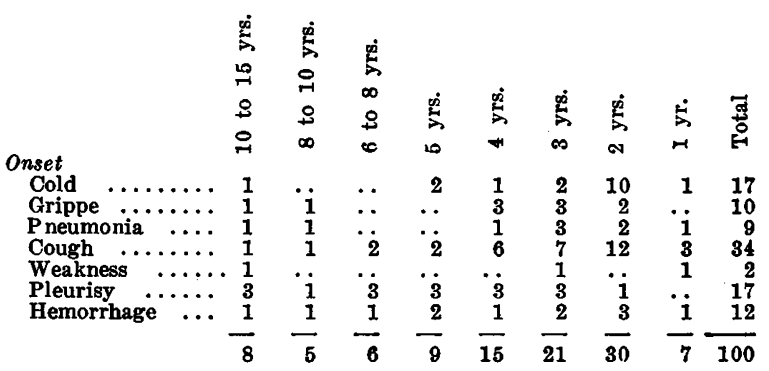

The salient points to be noted in comparing these groups are that, of the arrested cases, in only two was weakness the most prominent initial symptom, as compared with twenty-three patients in the group that died.

Marked weakness as an early symptom means poor resistance, and must be looked upon as of bad omen.

On the other hand, pleurisy and hemoptysis as . initial symptoms seem to be of favorable significance because their occurrence compels a patient's prompt attention and so he early becomes aware of active infection and the necessity for immediate treatment. 
TABLE 2.

Complications During Course of Disease.

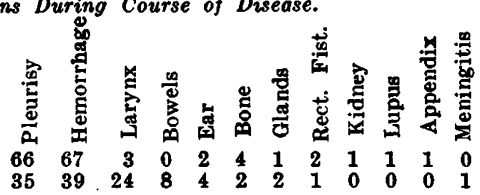

Pleurisy and hemoptysis during the course of the disease are also very frequent in the arrested cases. Pleurisy occurred in sixty-six of the favorable cases, as against thirty-five in the unfavorable group. Hemoptysis was present in sixty-seven of the arrested cases as compared with thirty-nine of the cases who died.

The conclusion that we draw from this table is that the pain from pleurisy and the hemorrhages may each be an indication of the production of antibodies in a sufficient amount to produce congestion and conditions favorable to healing.

Furthermore, the occasional occurrence of these symptoms prolongs proper treatment and we well know that the element of time is a large factor in determining ultimate results.

It is worth noting that tuberculous infections of the larynx were present in but three of the arrested cases, while twenty-four of the patients who died developed laryngitis, although the course of their disease was of shorter duration (average 14 months). This indicates that a low resistance must be the prime factor in the production of laryngitis, as the opportunity for contact infection is the same in all cases of open tuberculosis.

TABLE 3.

Age of Onset by Decades.

11 to 2021 to $30 \quad 31$ to $40 \quad 41$ to $50 \quad 51$ to 60

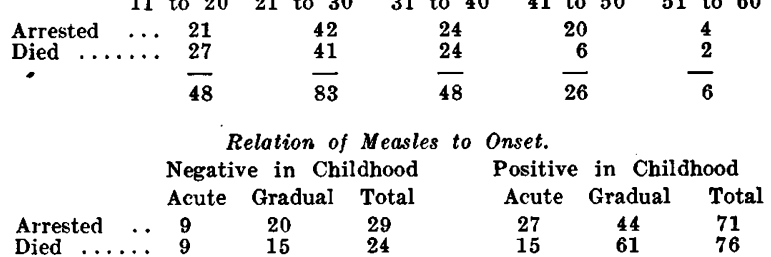

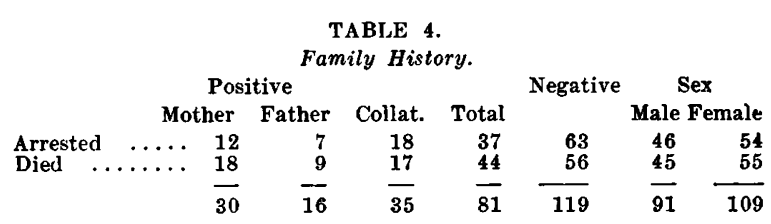

The family history was positive in thirtyseven of the arrested cases, as compared with forty-six of those who died. Although there are nine per cent. more favorable cases with positive family history, the difference is so small that one would not be justified in drawing conclusions that heredity influenced favorably or unfavorably the course of the disease.

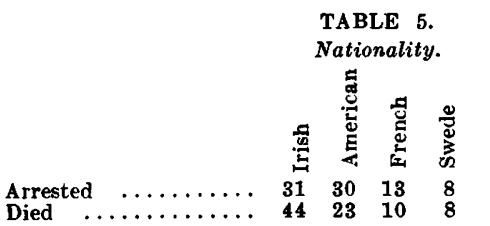

TABLE 6.

Sputum of Arrested Cases. Upon Admission. Upon Discharge. Bacilli of Sputum Bacilli Moderately advanced . Advanced ...........

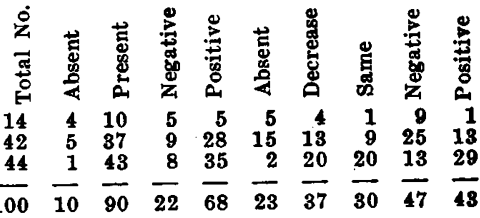

Note that in this table ten patients had no sputum on admission, although some of them were advanced cases. Twenty-three had no sputum on discharge. Sixty-eight had positive sputum on admission and forty-three on discharge.

We find that the amount of sputum corresponds with the activity of the disease. If the sputum increases in amount it indicates active tuberculous inflammation and as the quantity of sputum lessens the specimens which contain bacilli become fewer.

It required two examinations to find tubercle bacilli in the sputum of fifty patients, three examinations to find bacilli in the sputum of eighteen patients, four examinations to find bacilli in the sputum of eight patients, and five examinations to find bacilli in the sputum of three patients. During the year antiformin was used in testing specimens of sputum from sixty patients, seven of which proved positive, although bacilli could not be found by other methods.

This work shows the fallacy of putting dependence upon one, or more, negative examinations of sputum, even in cases of open tuberculosis.

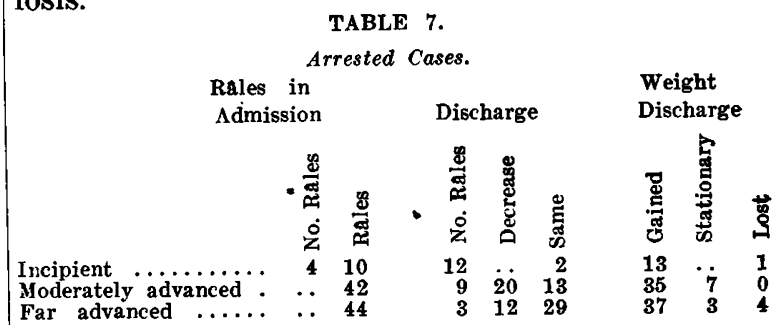

This table shows that all but four of the cases showed râles after cough. A much larger number failed to reveal râles on ordinary breathing. I wish to emphasize this point. Many diagnoses are delayed because examiners do not insist upon the patient making a short cough at the end of each expiration while auscultation is being done. Even if râles are heard on quiet breathing, this method will usually bring out râles over a larger area and often show other lobes involved. After râles once appear and the tissue cells are destroyed, comparatively few cases lose them, except after a long period of time.

In advanced cases râles sometimes disappear following fibroid changes, or the diseased area $\dot{q}$ softens, and being discharged leaves a cavity, with walls that have undergone firm cicatriza${ }_{5}^{2}$ tion. 
The presence of dry râles when considered alone, are of little importance in determining the activity or degree of arrest in an individual case.

The subjective symptoms are of greater value in considering prognosis, as well as in making an early diagnosis, because râles are of comparatively late appearance and remain after the active process heals.

Finally we will consider the pulse rate and temperature. Of the one hundred cases that obtained an arrest the average temperature for two weeks following admission was from 98.6 to 100. Eight others had a temperature of from 100 to 101 degrees. Forty-two had practically normal temperatures.

The pulse rate averaged from 90 to 105 in sixty-seven patients. The pulse was below 90 in all but twenty-eight, when diseharged.

When a patient's temperature does not remain under 99 after two weeks' rest in bed our experience teaches us that the prognosis for obtaining an arrested condition is extremely poor, and if the temperature remains elevated more than four weeks, that the disease will continue to progress in spite of sanatorium treatment.

The twenty-eight patients whose pulse rate was above 90 upon discharge have, in our opinion, a poor prospect of continuing to hold the degree of arrest obtained, or to further improve. The pulse rate seems to respond very early to the influence of tuberculous infection and remains the best indication of a patient's resistance throughout the course of the disease.

The points we wish to emphasize are the importance of watching for early subjective symptoms, as by them a diagnosis may be made before ulcerative processes occur to free the bacilli. Also, that the finding of bacilli depends much upon the persistence of the search made for them; and further, that tuberculosis, even in a.dvanced form, may be present without bacilli appearing in the sputum. Finally, in suspicious cases it is better to give patients the benefit of the doubt by telling them that their symptoms indicate early tuberculosis and to prevent further progress it will require a radical change in their mode of living. If a patient is sent to a sanatorium with a doubtful diagnosis no harm will have resulted, even if it should prove to be a simple bronchitis or an influenza infection.

The treatment is the same for the tuberculous and the nontuberculous and no harm has yet been known to result to a patient sent to a sanatorium even with a mistaken diagnosis, while irremediable harm results to many patients who are kept waiting for unmistakable symptoms of tuberculosis to develop.

I have purposely' omitted to consider in detail the signs to be found by physical examination because early changes are hard to detect except by specialists, whose field is limited to chest affections. The general practitioner is the one whom nearly all patients first consult. It is on his skill that most patients must rely for an early diagnosis. The family physician whose work is in all departments of medicine does not have the time to devote to careful chest examinations, or the constant practice which is necessary to note slight changes in lung tissue.

He does, however, have the opportunity to note subjective symptoms and his routine work tends to make him a keen observer. Physicians must recognize tuberculosis when it manifests itself by loss of weight, or strength, or a pulse of 90, or an elevated afternoon temperature, or blood spitting, or pleurisy, or a cough. Any combination of these symptoms which cannot be explained by other causes should be considered sufficient proof of active tuberculous infection.

Failure to make a positive diagnosis from these symptoms is too vital an error for a patient to forgive, but a mistaken positive diag. nosis leads to the right treatment, has no fatal sequel and will soon be forgotten.

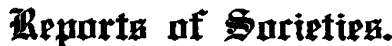

THIRTY-FOURTH ANNUAL MEETING OF THE AMERICAN LARYNGOLOGICAL ASSOCIATION.

$$
\text { First Day-Thursday, May } 9 t h \text {. }
$$

THE EXENTERATION OF THE ETHMOIDAL LABYRINTH BY THE INTRANASAL ROUTE.

Dr. Harris Peyton Mosher of Boston: The anterior boundary of the ethmoidal labyrinth is made by the internal angular process of the frontal bone and the posterior surface of the ascending process of the superior maxilla. The labyrinth cannot be entered effectively unless the curette is carried outward behind the ascending process of the superior maxilla toward the lachrymal bone. Removing the anterior end of the middle turbinate and curetting upward and not outward does not open the labyrinth to any extent. The internal angular process of the frontal bone makes two-thirds or the whole of the bony ring which is the first part of the nasofrontal duct. The best guide to the duct is the posterior surface of ascending process of the superior maxilla. In a large number of cases the nasofrontal duct is not a tubular canal but consists of a bony ring at the beginning and then becomes a triangular antero-posterior slit. This is more like an ethmoidal cell or an irregular meatus than a duct. The naso-frontal duct tends to run from without inward and to come into relationship with the extreme upper part of the anterior end of the middle turbinate. When the drainage canal of the frontal sinus has the cell form the anterior end of the middle turbinate makes its inner boundary. The duct, therefore, is reached most easily through the nose and through the anterior end of the middle turbinate at the level of the superior turbinate. A curette introduced at this point and carried outward toward the lachrymal bone and then withdrawn a little and carried straight downward and backward enters the anterior part of the labyrinth behind the ascending process of the superior maxilla and breaks down the cells through which the naso-frontal duct runs, destroying both the cells and the duct. Very little curetting is required to 